\title{
Desarrollo de competencias científicas mediante una unidad didáctica de ecosistemas en grado sexto
}

\section{Development of scientific competences through a didactic unit of ecosystems in a sixth grade}

DOI: http://dx.doi.org/10.17981/cultedusoc.11.2.2020.07

Recibido: 20 de enero de 2020 Aceptado: 07 de mayo de 2020 Publicado: 07 de julio de 2020

\author{
Jhorman Jesid Coronado-Peña \\ Universidad del Quindío. Arauca (Colombia) \\ coronadojhorman1995@gmail.com \\ Yeliza Builes-González (D) \\ Universidad del Quindío. Armenia (Colombia) \\ yelizabuiles1@gmail.com \\ Ángela Patricia Vargas-Orozco \\ Universidad del Quindío. Armenia (Colombia) \\ apvargaso@uqvirtual.edu.co \\ Nadia Lucía Obando-Correal \\ Universidad del Quindío. Armenia (Colombia) \\ nlobando@uniquindio.edu.co
}

Para citar este artículo:

Coronado-Peña, J., Builes-González, Y., Vargas-Orozco, Á. y Obando-Correal, N. (2020). Desarrollo de competencias científicas mediante una unidad didáctica de ecosistemas en grado sexto. Cultura, Educación y Sociedad, 11(2). 110-124. DOI: http://dx.doi. org/10.17981/cultedusoc.11.2.2020.07

\section{Resumen}

El artículo tiene como propósito presentar los resultados de una investigación orientada al fortalecimiento de competencias científicas en estudiantes mediante una unidad didáctica de ecosistemas en grado sexto. El estudio es de naturaleza mixta, aborda un componente cuantitativo por medio de la aplicación de un cuestionario a los estudiantes para validar conocimientos con relación al tema planteado; igualmente se trabaja un componente cualitativo a través de la Investigación Acción Participativa (IAP). Se exploran las ideas previas de los estudiantes con preguntas vinculadas al mundo de la vida, posteriormente se realiza una intervención didáctica que permita la conceptualización y construcción de saberes científicos. Finalmente se hace una valoración del proceso de aprendizaje mediante autoevaluación y heteroevaluación de lo conceptual, procedimental y actitudinal. Como resultado se evidencia el desarrollo de competencias del pensamiento científico como la observación de fenómenos particulares y su registro, formulación de hipótesis, análisis de resultados, generación de conclusiones y trabajo en equipo. Se concluye que las actividades experienciales permiten al estudiante ubicarse desde el rol de científico cuando lo invitan a que observe, registre y genere conclusiones que efectivamente promueven esas competencias del pensamiento científico.

Palabras clave: Pensamiento científico; factores bióticos; factores abióticos; mundo de la vida

\begin{abstract}
The purpose of the article is to present the results of research aimed at strengthening scientific skills in students through a didactic unit on ecosystems in sixth grade. The study is of a mixed nature, addressing a quantitative component through the application of a questionnaire to students to validate knowledge in relation to the topic raised, also working a qualitative component through the Participatory Action Research (PAR). The students' previous ideas are explored with questions linked to the world of life, and then a didactic intervention is carried out that allows the conceptualization and construction of scientific knowledge. Finally, an assessment of the learning process is made by means of self-assessment and heteroevaluation of the conceptual, procedural and attitudinal aspects. As a result, the development of scientific thinking skills is evident, such as the observation of particular phenomena and their recording, hypothesis formulation, analysis of results, generation of conclusions and teamwork. It is concluded that the experiential activities allow the student to place himself from the role of scientist when he is invited to observe, record and generate conclusions that effectively promote those competences of scientific thinking.
\end{abstract}

Keywords: Scientific thought; biotic factors; abiotic factors; world of life 


\section{INTRODUCCIÓN}

Según el Ministerio de Educación Nacional en los Estándares Básicos de Competencias en Ciencias Naturales (MEN, 2004), se reconoce que en un entorno cada vez más complejo, competitivo y cambiante; formar en Ciencias significa contribuir a la formación de ciudadanos capaces de razonar, debatir, producir y desarrollar al máximo su potencial creativo. Este desafío plantea la responsabilidad de promover una educación crítica, ética, tolerante con la diversidad y comprometida con el medio ambiente, que se constituya en puente para crear comunidades con lazos de solidaridad, sentido de pertenencia y responsabilidad frente a lo público y lo nacional. De este modo, es necesario crear condiciones para que los estudiantes aprendan sobre Ciencias Naturales y para que puedan comprenderlas y compartir sus experiencias y sus hallazgos, actuar con ellas en la vida real y hacer aportes a la construcción y al mejoramiento de su entorno; tal como lo hacen los científicos (p. 6).

No se trata de acumular conocimientos, sino aprender lo pertinente para la vida y lo aplicable para solucionar problemas nuevos en situaciones cotidianas. Se trata entonces de ser competente, no de competir. Hernández (2005) detalla que las competencias científicas son capacidades y disposiciones que influyen en el interactuar apropiadamente en situaciones en las cuales es necesario aplicar comprensiva los conocimientos.

Desde la perspectiva de la competencia científica, el estudiantado aborda estas cuestiones según su grado de comprensión de los conocimientos científicos, su capacidad para acceder a la información y para interpretar las pruebas científicas correspondientes. Todas ellas son capacidades cognitivas, pero, además, se toma en consideración la respuesta afectiva del alumnado, a través de aspectos relacionados con la actitud, el interés y la motivación ante las ciencias (Caño y Luna, 2011, p. 7).

Así, cuando se desarrollan procesos de enseñanza sobre temáticas en ciencias, resulta enriquecedor trabajarlos desde la cotidianidad, o en palabras de Husserl (2008), desde el mundo de la vida de cada estudiante, en el que cada uno habita (el cual es personal y contextualizado) y, que debe ser el punto de inicio y meta en los distintos aprendizajes.

Pese a lo anterior, en algunos países como Colombia aún se sigue educando de manera tradicional, donde por ejemplo, para la enseñanza de las ciencias se sigue privilegiando los métodos transmisivos que centran el proceso educativo de manera exclusiva en la memoria, de tal forma que no se incorporan aspectos relacionados con la curiosidad, formulación de preguntas, exploración, manipulación de objetos y experimentación; todos ellos necesarios para contribuir al desarrollo de competencias científicas (Ortiz y Cervantes, 2015; Eder y Osorio, 2018), indispensables para comprender y actuar en un mundo que cada vez está más permeado con avances científicos y tecnológicos.

En este sentido las prácticas transmisionistas siguen siendo usuales en las aulas de clase; tal es el caso la institución Inem José Celestino Mutis, Armenia, Colombia, donde los resultados en las pruebas Saber en el área de ciencias naturales, evidencian que más del $40 \%$ de los estudiantes, al ingresar al grado $6^{\circ}$, tienen dificultades para hacer uso del conocimiento científico, explicar fenómenos e indagar procesos propios de las ciencias naturales (ICFES, 2018). Además, los sujetos significantes seleccionados para la presente investigación, manifiestan el poco desarrollo de competencias de pensamiento científico 
que permitan y potencien lo que Sota (2015) plantea frente a la enseñanza de las ciencias: la curiosidad como instinto natural para que el estudiante se formule preguntas, explore, manipule y busque información para dar solución a un problema. Por lo anterior, el presente estudio buscó desarrollar competencias científicas mediante una unidad didáctica de ecosistemas en estudiantes del grado sexto de la Institución Educativa Inem José Celestino Mutis en Armenia-Quindío.

\section{Metodología}

El presente estudio se llevó a cabo mediante un proceso de investigación mixta que integra un componente cuantitativo y uno cualitativo; esto permite la validación de la información pues proporciona una perspectiva amplia y de gran alcance en los resultados, al aplicar estrategias básicas de integración (Pereira, 2011). La visión cuantitativa se desarrolla a través de la aplicación de un cuestionario a los estudiantes para validar conocimientos con relación al tema planteado; mientras que el componente cualitativo se cubre a través de la Investigación-Acción-Participativa (IAP); la integración de enfoques posibilita que la práctica educativa se convierta en suceso crítico de intervención y reflexión para formular alternativas de transformación de las prácticas colectivas (Vargas y Tatar, 2018). El diseño multimétodo a decir de Senior, Colina, Marín y Perozo (2012) representa un espacio metodológico apropiado para la convergencia disciplinar y fortalecimiento del pensamiento sistémico.

\section{Participantes}

Este estudio estuvo representado por 15 estudiantes de grado sexto con edades comprendidas entre los 12 y 14 años. Todos los estudiantes pertenecientes al plantel educativo Inem José Celestino Mutis; institución de carácter mixto, oficial, jornada diurna y ubicada en el área urbana de la ciudad de Armenia-Quindío, Colombia.

El criterio de selección de los sujetos significantes se debe a que, según los Derechos Básicos de Aprendizaje, los estudiantes a partir del grado tercero tienen sus primeros acercamientos con la ecología y los tópicos que aquí se pretenden trabajar, de modo que los educandos de grado sexto deben tener un conocimiento más elaborado sobre los mismos.

La unidad didáctica se construyó y aplicó en las fases que se enuncian a continuación:

\section{Momento 1: Exploración de ideas previas}

De acuerdo a las competencias establecidas en el plan de área de Ciencias Naturales del grado sexto en el cuarto periodo académico, los estudiantes debían realizar procesos que les permitieran identificar, analizar y describir la interacción entre los seres vivos y los factores abióticos de un ecosistema; articulando, además, el desarrollo de compromisos personales y sociales (participación y comunicación activa entre pares). Como primera medida se suministró a los estudiantes una prueba de indagación de ideas previas, basado en un cuestionario de 11 preguntas abiertas, ilustradas y vinculadas con el mundo de la vida; desde los conceptos que se esperaban abordar en la temática de ecosistemas. 


\section{Momento 2: Intervención}

En correspondencia con los resultados obtenidos a través del instrumento de ideas previas, se diseñaron tres fases que respondieran a las necesidades del grupo y permitieran el desarrollo de competencias científicas establecidas en los Estándares Básicos de Competencias emitidos por el MEN (2004), las cuales fueron: explorar hechos y fenómenos, analizar problemas, observar, recoger y organizar información relevante, utilizar diferentes métodos de análisis, evaluar los métodos, compartir los resultados, entre otros.

\section{Fase 1. Siembra de semillas "Proceso de fotosintesis"}

Con el fin que los estudiantes comprendieran las diversas dinámicas y procesos que ocurren en los ecosistemas se concibió inicialmente la aprehensión de procesos primordiales como la fotosíntesis, por medio de la siembra de frijoles dado que estos mantienen una dinámica de crecimiento rápido, además por la cercanía que los educandos tienen desde sus casas con dichas semillas. De esta manera, se suministraron semillas de frijoles para ser sembradas en recipientes que contenían sustrato abonado; con ello, los estudiantes hicieron las siembras en el huerto de la institución y realizaron registro de los cambios y observaciones ocurridas en el proceso teniendo en cuenta diversos suministros de agua (todos los días, día de por medio y ninguno de los días). Bajo esta dinámica, se planteó la pregunta: ¿qué frijoles germinarán primero?, de tal manera que los estudiantes propusieran hipótesis que permitieran resolver dicho interrogante.

Transcurrido cuatro días, los estudiantes socializaron la experiencia con base en lo registrado, realizaron cuestionamientos y confrontaron sus resultados en torno a la validez de las hipótesis formuladas inicialmente por ellos.

\section{Fase 2. Estudio de un ecosistema "la hojarasca"}

Durante esta fase se mostró a los estudiantes un video corto donde se narraban aspectos generales como la conformación y las diferencias entre los diferentes tipos de ecosistemas. A continuación, con la intención de hacer de la clase un proceso dinámico y experiencial; los docentes llevaron al aula lo siguiente: bandejas plásticas, hojarasca, pinzas de laboratorio, guantes, lupas y bolsas plásticas. Los estudiantes hicieron grupos de a tres personas, a los cuales se les proporcionó un kit con cada uno de los materiales antes mencionados; y debían hacer identificaciones, observaciones y descripciones en torno a organismos como plantas, animales y hongos encontrados en la hojarasca; esto con ayuda de una guía estructurada por los docentes la cual se denominó: "La vida en la hojarasca: un mundo de interacciones"; esta indicaba caracteres diagnósticos, hábitats y roles ecológicos de individuos que suelen hallarse en la hojarasca.

Seguidamente con el fin de que todos los estudiantes fueran partícipes del proceso, a cada equipo se le facilitó unas escarapelas que debían ser repartidas y apropiadas por cada estudiante, dado que indicaban roles específicos que debían de cumplir durante la experiencia. Para cerrar esta actividad, se dejó una tarea que constaba de preguntas orientadoras con el objetivo que contrastaran lo que aprendieron en clase con distintas fuentes de información. 
En la siguiente clase, en una mesa redonda se compartió y discutió con todos los compañeros lo investigado.

\section{Fase 3. Juego de roles "Cadenas y redes tróficas"}

Esta fase se llevó a cabo mediante un juego de roles en donde algunos estudiantes cumplieron el rol de organismos productores, consumidores y descomponedores y, otros el rol de factores abióticos como el agua, rocas, aire y luz. Ello se realizó usando escarapelas sobre ilustraciones y enunciados. Además, se dieron pequeñas intervenciones por los docentes para dar claridad en las temáticas.

Finalmente, los docentes construyeron un texto corto denominado "La vida: un mundo de interacciones; juntos funcionamos mejor" donde se describió brevemente los procesos vistos en clase. Este texto fue entregado a cada uno de los estudiantes y asimismo analizado en el aula.

\section{Momento 3: Valoración}

Este apartado se dividió en dos aspectos. En el primero el estudiante en su individualidad valoró sus conocimientos, actitudes y habilidades puestas en marcha, es decir, una autoevaluación; la cual comprendió de 4 ítems en el que se señalan aspectos particulares de las clases y donde había que reflexionar y colocar una puntuación de 1.0 a 5.0 según haya sido su compromiso en las clases. Por otro lado, se realizó una heteroevaluación que consistió en una serie de preguntas de selección múltiple acordes a lo tratado en el desarrollo de las clases; siempre tratado desde el mundo de la vida o con los intereses de estos.

Es importante mencionar que los instrumentos empleados en esta investigación durante la exploración de ideas previas, la intervención y la valoración, fueron evaluados y avalados por expertos en el tema; los cuales determinaron que los instrumentos reunían las condiciones de validación para su aplicación, al presentar concordancia y pertinencia con el objetivo propuesto en la investigación.

\section{Resultados y Discusión}

\section{Exploración de ideas previas}

Se realizó un proceso inductivo en el que se agruparon los datos a partir de las coincidencias entre las respuestas de los participantes, asociándose según las repeticiones o a las que fueron tendencia. Una vez realizado el conteo de dichas categorías, se calcularon porcentajes y se hizo el respectivo análisis e interpretación de los datos obtenidos (Rojas, Vargas y Obando, 2017) y se encontró lo siguiente:

Mediante la primera pregunta descrita en el instrumento diagnóstico se buscó cuestionar el concepto de ecosistema, a través del interrogante: ¿qué crees que es un ecosistema?, encontrando que el $46 \%$ lo describe como un conjunto de seres vivos y no vivos que habitan en un lugar determinado. Así, entre los elementos mencionados reiteradamente se encontraron las plantas y los animales, dejando a un lado la presencia de otros organismos como los hongos, las bacterias, las algas, entre otros; mientras que, de los factores abióticos, los 
estudiantes mencionaron otros elementos como la luz, el agua y el aire. Asimismo, el 27\% de los sujetos significantes expresaron que estos se referían exclusivamente a un conjunto de seres vivos, ignorando los factores abióticos, mientras que otro $27 \%$ no respondió la pregunta.

Respecto a lo anterior, aunque, la mayoría de los estudiantes entiende el ecosistema como el resultado de la interacción entre factores abióticos y bióticos, en la descripción de sus componentes se destaca la prevalencia de los animales y de las plantas (Bell-Basca, Grotzer, Donis \& Shaw, 2000), donde la importancia dada a estos dos reinos se debe principalmente al carácter utilitario que se les da (Rincón, Medellín y Vargas, 2004). Estas representaciones próximas a la experiencia de los estudiantes excluyen la función e importancia del resto de organismos; no obstante, algunos autores (Leach, Driver, Scott \& Wood-Robinson, 1996; Grotzer y Basca, 2003) coinciden en que muchos estudiantes no reconocen el papel de otros seres vivos como los microorganismos; por ejemplo, en los procesos de descomposición de la materia orgánica, ciclos de nutrientes, pues mantienen ideas negativas centradas en la amenaza que suponen para la salud (Faccio et al., 2013) desconociendo así su distribución y su papel en el mantenimiento en los ecosistemas.

A través de la pregunta dos: ¿será tu casa y el colegio un ecosistema?, se determinó que el 40\% de los estudiantes considera la casa y el colegio un ecosistema argumentando que allí normalmente se encuentran con los elementos que lo conforman. Sin embargo, un $27 \%$ señala que no es posible que la casa y el colegio sea un ecosistema porque no hay vegetación ni animales (Figura 1), a pesar de que la institución dispone de buenas coberturas de vegetación e incluso, en la parte trasera del aula se encuentra una huerta escolar.

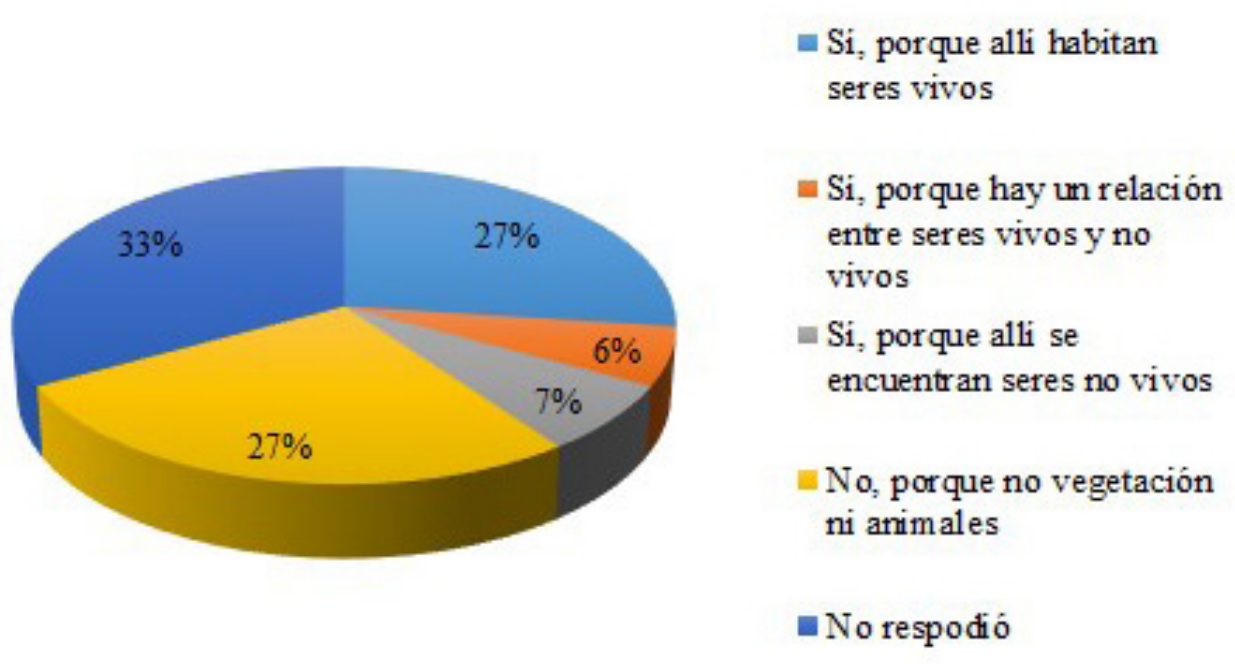

Figura 1. ¿Será tu casa y colegio un ecosistema?

Fuente: Elaboración propia.

En vista de considerar las construcciones humanas (como las casas y la escuela) parte de los ecosistemas, Zarrilli (2000) manifiesta que los estudiantes mantienen una condición sagrada, mística, y aislada del ambiente, de manera que generan dos interpretaciones del medio completamente separadas y diferentes; por un lado se asocia a los ecosistemas únicamente con el medio rural o natural, carente de intervención humana y donde la única condición para que funcionen adecuadamente es la ausencia del hombre en dichos espacios; y por el otro se excluye a la ciudad considerándola como una construcción artificial 
que difícilmente puede asociarse a lo natural, como muestra de la visión fragmentada y reduccionista que de ambiente y ecosistema manejan los estudiantes. Al consultarse a los estudiantes con relación a la concepción de la casa y la escuela como un ecosistema los resultados se evidencian en la Figura 1.

Por otro lado, ante el interrogante: ¿será que el agua, el suelo, el aire, las rocas y la luz hacen parte de un ecosistema? (factores abióticos en el ecosistema), se pudo apreciar que el 80\% de los estudiantes determinan estos factores como factores abióticos que hacen parte de un ecosistema; aunque estos factores no representan algo vivo, los estudiantes tienen presente que hacen parte de un ecosistema y que cumple una función determinada. El restante de estudiantes (20\%) desconoce la situación, por lo que no respondieron.

De modo similar, Fernández y Casal (1995) determinaron que los factores abióticos más mencionados son el agua, luz y temperatura, señalando que son imprescindibles para la vida, sin embargo, no logran describir de qué manera se relacionan con los seres vivos y cuál es la importancia de esta interacción dentro de los ecosistemas (p.300). Esta situación se ha determinado previamente como una dificultad generalizada, pues en un análisis realizado por Eilam (2002), los componentes abióticos no son muy tenidos en cuenta y difícilmente se establecen interacciones con los componentes bióticos como lo es la importancia de la luz solar para los procesos de crecimiento y desarrollo de las plantas (Driver, Squires, Rushworth y Wood-Robinson, 1994). Se infiere de lo planteado la importancia del desarrollo científico en el estudiante; al respecto Zambrano et al. (2018), resaltan la importancia de la investigación como una estrategia formativa y pedagógica que conlleva al desarrollo de competencias de indagación y descubrimeinto.

Aunque en el inicio de la exploración de ideas previas la mayoría de los estudiantes sólo dimensiona a las plantas y animales como parte de un ecosistema, para el caso de la pregunta 4: ¿será que los animales, las plantas, las bacterias, los hongos y las algas, los cuales son factores bióticos vivos, hacen parte de un ecosistema? (composición biótica de un ecosistema), el 93\% de los estudiantes presentan una idea más amplia al relacionar también a las bacterias, los hongos y las algas como factores bióticos que están inmersos en un ecosistema. No obstante, puede que esta respuesta sea un poco sesgada debido a que en la pregunta se relaciona a estos organismos. Dentro de esta respuesta los estudiantes dan nociones respecto a la dinámica de cadenas y redes tróficas que se presentan en los ecosistemas, puesto que, indican que estos individuos se encuentran en un ecosistema porque es necesario para la vida; nos ayudan a vivir, es esencial para el mantenimiento de todos los seres vivos. Los demás encuestados no respondieron a este interrogante.

Se consultó a los estudiantes acerca de las necesidades de las plantas para crecer (interrogante 5: ¿factores abióticos? ¿factores bióticos?), la mayoría (93\%) señalan dentro de las condiciones para el crecimiento de las plantas es indispensable seres vivos y factores abióticos como agua, aire, tierra y la luz; esto permite el desarrollo; crecimiento y la vida de la mismas. El resto de los estudiantes no respondió a la pregunta.

El interrogante 6 señalaba: ¿qué piensas de la dinámica de una vaca alimentándose de pasto? (alimentación en el ecosistema), la mayoría (93\%) describe que las vacas se alimentan del pasto porque esto es necesario para sobrevivir (así como el ser humano necesita alimento para vivir, ellos también lo requieren). Por otro lado, una postura más particular en cuanto al desarrollo de la respuesta a esta pregunta, la describen algunos estudiantes 
(7\%) y es que, señalan que esto hace parte de la alimentación de la vaca y es fundamental para la reproducción.

La pregunta 7 describió: ¿es necesario en el ecosistema que una especie animal se alimente de otro? Por ello se mostraba a un animal alimentándose de otro. Al respecto el 93\% de los estudiantes señalan que es indispensable esto dentro del ecosistema puesto que todos los animales hacen parte de una cadena alimenticia y ello es parte del ciclo de la vida, y, además, en ocasiones esto permite el control de las plagas. Otra respuesta muy interesante a esta pregunta se articula con la percepción frente a la estética de los animales en el momento de la alimentación, pues para el 7\% de los estudiantes no es necesario que los animales se alimentan entre sí porque estéticamente no es agradable.

Con relación a si al desayunar, almorzar y cenar se hace parte de la cadena alimenticia (pregunta 8), el 100\% de los estudiantes indicaron que sí; porque es necesario para vivir, el crecimiento, y además, porque somos parte de la cadena alimenticia.

Frente a la pregunta 9, sobre qué creen los estudiantes con relación al crecimiento de algunas plantas en espacios no muy adecuados para ellas, el 33\% expresan que en el sitio en donde se encuentren las plantas siempre encontrarán la forma para obtener los recursos necesarios de sobrevivencia (indicando así percepción en torno a la adaptación) (Figura 2). Siendo así, dicha percepción sobre "adaptación" a ambientes adversos, vista como un mecanismo que permite la supervivencia de las plantas bajo diferentes condiciones; aunque ciertas situaciones pueden no ser óptimas en todos los casos, por el momento, les permiten sobrevivir ante las presiones ambientales y asegurar su prevalencia en el medio (Gándara, Gil y Sanmartí, 2002).

Desde otra perspectiva, un $27 \%$ de los participantes detallan que las plantas pueden realizar estos procesos porque pueden vivir en cualquier lugar (Figura 2) (aquí se puede percibir nociones de los estudiantes respecto a que las especies vegetales podrían ser organismos cosmopolitas).
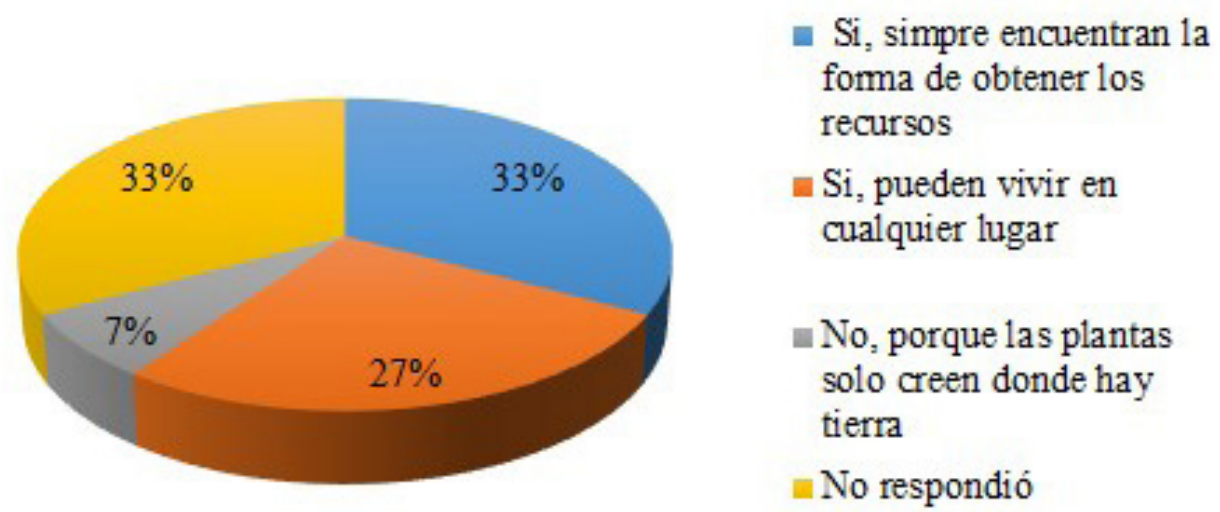

Figura 2. ¿Crees que algunas plantas pueden crecer en lugares no tan adecuados para ellas?, por ejemplo, en tierras áridas como el desierto o en las calles cementadas de la ciudad. Fuente: Elaboración propia.

Por su parte, respecto a la competencia en los ecosistemas (pregunta 10: algunos organismos como las plantas y animales compiten por sus recursos con otros ¿sucede esto en todos los ecosistemas?) un gran porcentaje de los estudiantes (86\%) consideran necesario que los seres vivos compitan por recursos, y, además, esta dinámica es fundamental en todos los 
ecosistemas porque allí se lucha por la supervivencia. El restante de sujetos significantes no dio respuesta a la pregunta.

En este estudio, al igual que lo descrito por Cepeda, Martínez y Rangel (2016) se puede evidenciar que los estudiantes comprenden las dinámicas alimentarias como un flujo de energía lineal, desconociendo la red que establecen las interacciones complejas entre organismos de un mismo ecosistema.

En la última pregunta se consultó si la huerta del colegio era un ecosistema, nuevamente los estudiantes relacionaron las plantas con los ecosistemas, por cuanto el $93 \%$ concibe a la huerta como un ecosistema, allí crecen plantas, proveen oxígeno, sirven de alimento y de empleo medicinal. Por otro lado, en algunos estudiantes (7\%) no se concibe la huerta como un ecosistema en vista que no hay animales.

Las ideas previas suelen ser entendidas como aquellas concepciones acerca de un pensamiento propio de la experiencia previa a la enseñanza y surgen por interacción con la vida cotidiana (Gándara et al., 2002). Las cuales más allá de ser un simple diagnóstico del estado de conocimiento, permiten el desarrollo conceptual en los procesos de enseñanza y aprendizaje. Por tanto, en esta investigación se considera la indagación de los conocimientos iniciales de los estudiantes como elemento de reflexión para la planeación de clase; de esta forma, facilita al docente en el proceso determinar los conceptos a transferir posteriormente al aula. Además, permite al acceso al conocimiento, manejo y apropiación de las competencias científicas de los estudiantes; al respecto se evidenció que se limitan a repetir conceptos y, no suelen plantear hipótesis como base para el análisis crítico y coherente del contexto.

\section{Intervención}

Durante el proceso de siembra de semillas de frijol, los estudiantes no solo se apropiaron de conceptos y dinámicas específicas como el de la fotosíntesis y el de los factores tanto abióticos como bióticos, esta fase 1 también permitió trabajar en los estudiantes el desarrollo de competencias científicas, esto de la siguiente forma: observación de fenómenos específicos en el momento que los estudiantes vivenciaron gran parte del proceso de desarrollo y crecimiento de una especie vegetal en particular, de igual manera, allí los estudiantes pudieron identificar ciertas condiciones influyentes en los resultados, en la medida que observaron lo ocurrido a las especies al suministrar o no agua.

Otro aspecto importante, es que los mismos estudiantes pudieron plantear sus hipótesis para después corroborarlas, confrontarlas o discutirlas; esto resultó influyente en el proceso de enseñanza y aprendizaje, se puede ver la educación como todo un proceso de construcciones donde los mismos estudiantes ven la necesidad de modificar o reestructurar sus conocimientos. Es así como el papel docente empieza a ser de mediador y deja de un lado la noción de transmisor de conceptos.

Asimismo, se percibió el interés de los estudiantes en el momento de hacer registros de sus observaciones, por cuanto sus procesos no solo eran compartidos por el docente, sino que ellos mismos podían experimentarlos. De esta manera, es necesario para el docente construir sus escenarios donde los estudiantes puedan aproximarse a la dinámica de la metodología científica; al analizar por ejemplo con rigor los "requerimientos de una especie vegetal", situándose así al estudiante bajo el rol de científico. 
Con respecto a la segunda fase (estudio de un ecosistema "la hojarasca"), se encontró que los estudiantes concentraron su interés durante el desarrollo donde pudieron explorar, observar, analizar hechos y fenómenos; se crea un ambiente propicio para el aprendizaje para desplegar y estructurar competencias científicas. Asimismo, pudo verse el aula de clase como un sitio de experimentación y por consiguiente, comprender los procesos científicos como hechos cotidianos y no como sucesos inalcanzables e imposibles de comprender. Un suceso importante, fue el empleo de accesorios tecnológicos como la lupa y las pinzas de laboratorio que permitieron a los estudiantes estar inmersos con dichos materiales.

Otro instrumento valioso en la segunda fase lo representó la guía construida por los docentes, fue bastante gráfica y de fácil comprensión; permitió reforzar conocimientos respecto a los organismos hallados en la hojarasca. Del mismo modo, dado que los docentes habían identificado el rechazo de los estudiantes por trabajar en equipos, mediante el uso de las escarapelas y asignaciones de roles, se pudo fomentar actitudes positivas hacia dichas habilidades sociales. También la guía conllevó a los estudiantes a hacer observaciones más específicas de acuerdo a lo que se quería estudiar.

Igualmente, esta fase incluyó un proceso de investigación en otras fuentes de información, bien fuera en internet, libros impresos, entre otros; esto resultó agradable para los estudiantes, ya que allí encontraron diferencias y similitudes con lo que habían construido en el aula con los docentes; se consolida el pensamiento crítico a través de sucesos y argumentos convincentes. En este sentido, las actividades ofrecen al alumno la oportunidad de especular y explorar; esto permite el trabajo con otros para que puedan comunicar y escuchar ideas, así como establecer posturas críticas y reflexivas (Gadanidis, 1994) posibilitando la construcción colectiva del aprendizaje.

En la última fase del segundo momento se desarrollaron simulaciones respecto a funciones tanto de seres vivos como de factores abióticos; se logró la comprensión de temáticas como las cadenas y redes tróficas dadas en los ecosistemas. Es entonces como pese al grado de escolaridad de dichos estudiantes, se sigue considerando relevante utilizar el juego en procesos de enseñanza-aprendizaje; el estudiante explora diversas situaciones a partir de un rol particular; puede analizar problemas mediante la misma observación del entorno, recoger y organizar información relevante para solucionar situaciones problemas y fortalecer su pensamiento científico.

Finalmente, la implementación de textos cortos con imágenes, se consideró como otro mecanismo que coadyuvó en la construcción de conocimientos, puesto que mediante estos se pudo consolidar características frente a las temáticas y que también podrían incidir positivamente en los procesos de valoración.

La enseñanza de las ciencias en la actualidad representa una serie de retos y desafíos para los docentes, ya que como lo menciona Camacho (2013), la enseñanza científica se ha limitado a la presentación de conocimientos elaborados, sin permitir oportunidades para que los estudiantes se involucren y vivencien procesos y fenómenos característicos de la actividad científica, influenciando negativamente la enseñanza. A este respecto, autores como Pérez y Ochoa (2017) mencionan alternativas como la Escuela Activa, donde se propone la acción como condición del aprendizaje, y la experiencia como el mecanismo que permite a los alumnos reflexionar y apropiarse de los conocimientos; su objetivo es preparar a los estudiantes para la vida a través del desarrollo de sus capacidades, de la autoconstrucción 
de los conocimientos y de la experiencia inmediata con el medio en el que habitan con la intención de transformarlo (De Zubiría, 2006).

\section{Valoración}

En cuanto al primer aspecto de valoración que fue el individual (autoevaluación), representa un proceso importante por cuanto no solo docentes deben evaluar el desempeño de los estudiantes, sino que los mismos estudiantes pueden reflexionar sobre aspectos positivos o de mejora frente a su rendimiento académico y personal.

Igualmente se implementó la heteroevaluación además de servirle al docente para corroborar algunos logros del curso, se planteó con la finalidad de ejercitar y preparar a los estudiantes para las pruebas censales de $9^{\circ}$ y $11^{\circ}$. Esta evaluación estuvo enfocada a situaciones problema relacionados con el mundo de la vida de los estudiantes; la cotidianidad, los intereses y con los temas abarcados en el desarrollo de la temática.

Con relación a ello, dentro de los elementos esenciales para un plan de aula y/o preparación de una clase, se resalta la planeación y desarrollo de instrumentos para la evaluación de avances y desempeños en torno a los aprendizajes esperados. Es preciso mencionar que en los últimos años se ha transitado de una evaluación como sinónimo de medición, donde el énfasis estaba puesto en la dimensión instrumental (el cómo de la evaluación), para lograr una medición objetiva y científica del rendimiento escolar; hacia una perspectiva de la evaluación mucho más amplia y compleja, no restringida a su dimensión técnica, y que incluye conceptos como justicia, equidad y emancipación (Moreno, 2016, p. 53).

Como lo plantea este último autor, en el actual escenario la evaluación del aprendizaje, es adaptada y contextualizada; orientada a fomentar el desarrollo competencias de los alumnos, no para que memoricen datos sino para comprenderlos. En el estudio se diseñaron diversos instrumentos y actividades con las cuales se buscó potenciar el desarrollo de competencias específicas como Identificar, Indagar y Explicar, que corresponden a capacidades asociadas a la formación en ciencias y son evaluadas en las pruebas SABER (Mancilla, Altamirano, Estrada y Paucar, 2011).

En este sentido, los resultados de la presente investigación develan que lo empírico traza caminos hacia la formación científica de los estudiantes. Por ello, es fundamental promover la experimentación continua en el aula que conlleve a los estudiantes a hacer inferencias sobre sus acciones y sobre las acciones de los demás. De esa manera, se obtendrán evidencias que contribuirán a aprender, explorando relaciones causales y poniendo a prueba distintas ideas acerca de cómo funciona el mundo. Así, el involucrar a los niños en investigaciones y exploraciones acerca de los fenómenos de la naturaleza como forma de construir las bases del pensamiento científico, en tanto este enfoque didáctico va de la mano del modo en que espontáneamente se comienza a explorar el mundo (Furman, 2016).

Metz (1998) enfatiza el valor de brindarles a los niños oportunidades sostenidas de participación en prácticas científicas, poniendo el acento tanto en la experimentación como en el intercambio y la revisión de ideas, en el marco de la comunidad de aprendizaje del aula, como manera de potenciar y profundizar sus capacidades de pensamiento científico. De modo que, como docentes se está llamado a crear espacios que estimulen la observación, generación de hipótesis, experimentación y la exploración del medio para la comprensión de conceptos básicos en la formación como el de ecosistemas (p. 181). 


\section{Conclusiones}

El poner en ejercicio actividades como la siembra de semillas en diferentes condiciones, la observación de hojarasca, y el juego de roles de cadenas y redes tróficas, son alternativas que a partir de contenidos como "los ecosistemas", permiten desarrollar competencias científicas en los estudiantes, ofreciendo ventajas como su fácil diseño, bajos costos y la vinculación y participación de todos los estudiantes.

Por otro lado, la experimentación en el aula despierta el interés de los estudiantes por cuanto contribuye a la elaboración de ideas, nociones, habilidades, actitudes y sentimientos respecto a la ciencia; y los convierte en sujetos activos durante su proceso de aprendizaje; donde además convergen aspectos de carácter social.

Las actividades experienciales conllevan al estudiante a ubicarse desde el rol de científico cuando lo invitan a que observe, registre y genere conclusiones, efectivamente promueven esas competencias del pensamiento científico. Además, el estudiante considera la ciencia como un hecho en comunidades que se valida a través de diferentes formas (oral y escrita), y lo más importante que es un proceso donde cabe la crítica, el debate, las distintas posiciones frente a un aspecto o suceso a estudiar.

Los estudiantes vivenciaron un escenario para hacer ciencia escolar, la cual pudo encaminar a comprender aspectos clave de la ciencia; dejando a un lado la idea de que el hacer ciencia sólo es posible en los laboratorios convencionales. Así, se recomienda que las temáticas en ciencias se aborden bajo trabajos experimentales, pues la experimentación traza caminos hacia la formación científica de los estudiantes.

\section{AgRAdecimientos}

Se agradece a la Institución Educativa Inem José Celestino Mutis de Armenia-Quindío, por permitir los espacios y tiempos para el desarrollo y culminación del estudio. Asimismo, se da gracias a la disposición de los administrativos, docentes y estudiantes que posibilitaron y facilitaron el proceso de la práctica.

\section{REFERENCIAS}

Bell-Basca, B., Grotzer, T.A., Donis, K. \& Shaw, S. (May, 2000). Using domino and relational causality to analyze ecosystems: Realizing what goes around comes around. Paper presented National Association of Research in science Teaching, NARST, New Orleans, USA, pp. 1-28. Recuperado de http://citeseerx.ist.psu.edu/viewdoc/download? doi=10.1.1.34.8052\&rep=rep1\&type $=$ pdf

Camacho, J. P. (2013). Concepciones sobre ciencia y género en el profesorado de química: aproximaciones desde un estudio colectivo de casos. Ciência \& Educação, Bauru, 19(2), 323-338. Recuperado de http://www.nutes.ufrj.br/abrapec/viiienpec/resumos/R0117-1. pdf

Caño, A. y Luna, L. (2011). Pisa: competencia científica para el mundo del mañana. Bilbao: Instituto Vasco de Evaluación e Investigación Educativa. Asturias: ISEI-IVEI, OECD. PISA. Recuperado de http://iesmardearagon.es/documentos/ciencias_pisa2009completo. pdf 
Cepeda, W., Martínez, M. y Rangel, M. (2016). Comprensión del concepto de red trófica y su diferencia con la cadena trófica mediante trabajos prácticos y preguntas conflicto para tres ecosistemas colombianos. Bio-Grafía Escritos sobre la Biología y su Enseñanza, (Número extraordinario), 1712-1720. https://doi.org/10.17227/20271034.vol.0num.0biografia1712.1720

De Zubiría, J. (2006). Los modelos pedagógicos. Hacia una pedagogía dialogante. Bogotá, D.C.: Cooperativa Editorial Magisterio. Recuperado en https://www.institutomerani. edu.co/noticias/hacia-una-pedagogia-dialogante.pdf

Eder, L. Y. y Osorio, L. D. (2018). El desarrollo de pensamiento crítico en ciencias naturales con estudiantes de básica secundaria en una Institución Educativa de Pereira - Risaralda. Diálogos sobre educación, 9(16), 1-24. https://doi.org/10.32870/dse.v0i16.400

Driver, R., Squires A., Rushworth, P. y Wood-Robinson, V. (1994). Nutrition. In: R. Driver, A. Squires, P. Rushworth \& V. Wood-Robinson (Ed), Making Sense of Secondary Science: Research into Children's Ideas, (pp. 27-35). New York: Routledge.

Eilam, B. (2002). Strata of comprehending ecology: Looking through the prism of feeding relations. Science Education, 86(5), 645-671. https://doi.org/10.1002/sce.10041

Faccio, E., Costa, N., Losasso, C., Cappa, V., Mantovani, C., Cibin, V., Andrighetto, I. \& Ricci, A. (2013). What programs work to promote health for children? Exploring beliefs on microorganisms and on food safety control behavior in primary schools. Food control, 33(2), 320-329. https://doi.org/10.1016/j.foodcont.2013.03.005

Fernández, R. y Casal, M. (1995). La enseñanza de la ecología: Un objetivo de la educación ambiental. Enseñanza de las ciencias, 13(3), 295-311. Recuperado de https://www. raco.cat/index.php/Ensenanza/article/view/21419

Furman, M. (2016). Educar mentes curiosas: la formación del pensamiento científico y tecnológico en la infancia. Buenos Aires: Santillana.

Gadanidis, G. (1994). Deconstructing Constructivism. The Mathematics Teacher, 87(2), 91-94. Recuperado de https://www.jstor.org/stable/27968738

Gándara, M., Gil, M. y Sanmartí, N. (2002). Del modelo científico de «adaptación biológica» al modelo de "adaptación biológica» en los libros de texto de Enseñanza Secundaria Obligatoria. Enseñanza de las ciencias, 20(2), 303-314. Recuperado de https://ddd.uab. cat/pub/edlc/02124521v20n2/02124521v20n2p303.pdf

Grotzer, T. A. y Basca, B. B. (2003). How does grasping the underlying causal structures of ecosystems impact students' understanding? Journal of Biological Education, 38(1), 16-29. https://doi.org/10.1080/00219266.2003.9655891

Hernández, C. (2005). ¿Qué son las competencias científicas? Ponencia presentada en el Foro Educativo Nacional 2005, Ministerio de Educación, Madrid, España. Recuperado de http://www.acofacien.org/images/files/ENCUENTROS/DIRECTORES_DE_ CARRERA/I_REUNION_DE_DIRECTORES_DE_CARRERA/ba37e1_QUE\%20 SON\%20LAS\%20COMPETENCIAS\%20CIENTIFICAS\%20-\%20C.A.\%20Hernandez.PDF

Husserl, E. (2008). La crisis de las ciencias europeas y la fenomenología trascendental. Buenos Aires: Prometeo. Recuperado de https://profesorvargasguillen.files.wordpress. com/2013/08/crisis-de-las-ciencias-europeas-y-la-fenomenologc3ada-trascendentaltrad-julia-iribarne-krisis.pdf 
ICFES. (2018). Publicación de resultados Saber $3^{\circ}, 5^{\circ}$ y $9^{\circ}$. [Online]. Recuperado de: http:// www2.icfesinteractivo.gov.co/ReportesSaber359/seleccionListaInstituciones.jspx

Leach, J., Driver, R., Scott, P. \& Wood-Robinson, C. (1996). Children's ideas about ecology 3 : ideas found in children aged 5-16 about the interdependency of organisms. International JournalofScienceEducation, 18(2), 129-141.https://doi.org/10.1080/0950069960180201

Mancilla, H., Altamirano, A., Estrada, A. y Paucar, J. (2011). Especialización: Ciencia y Ambiente. Lima: PRONAFCAP.

Metz, K. (1998). Scientific inquiry within reach of young children. London: Springer.

Moreno, T. (2016). Evaluación del aprendizaje y para el aprendizaje: reinventar la evaluación en el aula / Tiburcio Moreno Olivos. México, D.F.: UAM, Unidad Cuajimalpa. Recuperado de http://www.casadelibrosabiertos.uam.mx/contenido/contenido/Libroelectronico/Evaluacion_del_aprendizaje_.pdf

Ortiz, R. y Cervantes, M. (2015). La formación científica en los primeros años de escolaridad. Panorama, 9(17), 10-23. Recuperado de https://journal.poligran.edu.co/index.php/ panorama/article/view/788/578

Pereira, P. Z. (2011). Los diseños de método mixto en la investigación en educación: Una experiencia concreta. Educare, XV(1), 15-29. Recuperado de https://www.revistas.una. ac.cr/index.php/EDUCARE/article/download/867/15910/

Pérez, L. y Ochoa, A. (2017). La participación de los estudiantes en una escuela secundaria. Retos y posibilidades para la formación ciudadana. Revista Mexicana de Investigación Educativa, 22(72), 179-207. Disponible en http:/www.comie.org.mx/revista/ v2018/rmie/index.php/nrmie/article/view/9

República de Colombia. MEN. (2004). Estándares Básicos de Competencias en Ciencias Naturales y Ciencias Sociales. [Online]. Recuperado de https://www.mineducacion.gov. co/1759/articles-81033_archivo_pdf.pdf

Rincón, M. E., Medellín, F. y Vargas, C. (2004). Concepciones sobre nociones ecológicas en niños de las escuelas rurales de Villeta (Cundinamarca). Bogotá, D.C.: CIUP.

Rojas, A. V., Vargas, O. A. P. y Obando, C. N. L. (2017). Concepciones sobre la enseñanza y aprendizaje de las ciencias naturales en el grado tercero de una institución educativa oficial del municipio de Calarcá Quindío. Revista de la Asociación Colombiana de Ciencias Biológicas, 1(29), 119-132. Recuperado de https://revistaaccb.org/r/index.php/ accb/article/view/143

Senior, A., Colina, J., Marín, F. y Perozo, B. (2012). Visión complementaria entre los métodos cualitativos y cuantitativos en la investigación social. Una aproximación teórica. Multiciencias, 12(Extra), 106-114. Disponible en http://produccioncientificaluz.org/index.php/multiciencias/article/view/19006

Sota, M. L. (2015). Experimentos sencillos para el desarrollo de la actitud científica en los estudiantes de cinco años de la Cuna Jardín Nº3. Huaral-2015. [Tesis de maestría]. Universidad Peruana Cayetano Heredia, Lima, Perú. Recuperado de http://repositorio.upch.edu.pe/bitstream/handle/upch/257/Experimentos.sencillos.para.el.desarrollo. de.la.actitud.cient\%C3\%ADfica.en.los.estudiantes.de.cinco.a\%C3\%B1os.de.la.Cuna. Jard\%C3\%ADn.N\%C2\%BA.03.Huaral-2015.pdf?sequence=3\&isAllowed=y 
Vargas, F. Y. y Tatar, J. E. (2018). La investigación acción participante: una oportunidad para la transformación de la cátedra de paz en la universidad. Revista Ciudad Pazando, 10(2), 40-53. https://doi.org/10.14483/2422278X.12716

Zambrano-Quintero, Y., Rocha -Roja, C., Flórez-Vanegas, G., Nieto-Montaño, L., JiménezJiménez, J. y Núñez-Samnández, L. (2018). La huerta escolar como estrategia pedagógica para fortalecer el aprendizaje. Cultura. Educación y Sociedad 9(3), 457-464. https://doi.org/10.17981/cultedusoc.9.3.2018.53

Zarrilli, A. G. (2000). Reseña de "Ambiente y sociedad. Conceptos y relaciones" de Carlos Reboratti. Mundo Agrario, 1(1), 1-3. Recuperado de http://www.memoria.fahce.unlp. edu.ar/art_revistas/pr.630/pr.630.pdf

Jhorman Jesid Coronado Peña es coordinador y docente de la Fundación social y educativa EDUCAR (Colombia). Licenciado en Biología y Educación Ambiental de la Universidad del Quindío. Mejor Puntaje de Calidad en el Segundo Periodo Académico 2018. Programa Licenciatura en Biología y Educación Ambiental, Universidad del Quindío (Mayo de 2019). Mejor Puntaje de Calidad en el Segundo Periodo Académico 2017. Programa Licenciatura en Biología y Educación Ambiental, universidad del Quindío (Mayo de 2018). Mejor Puntaje de Calidad en el Primer Periodo Académico 2016. Programa Licenciatura en Biología y Educación Ambiental, Universidad del Quindío (Diciembre de 2016). Mejor aprendiz 2013 del Servicio nacional de aprendizaje-SENA (Arauca, Colombia). https://orcid. org/0000-0002-8554-8088

Yeliza Builes González es licenciada en Biología y Educación Ambiental. Diplomado en Actualización de la Enseñanza de las Ciencias Naturales. Con intereses en la enseñanza en los distintos niveles académicos, en especial en la escuela primaria. https://orcid.org/00000002-7674-481

Ángela Patricia Vargas Orozco es licenciada en Biología y Educación Ambiental. https:// orcid.org/0000-0002-0170-3424

Nadia Lucía Obando-Correal es profesora de la Universidad del Quindío (Colombia). Magíster en Educación de la Universidad Tecnológica de Pereira (Colombia). Doctoranda en Didáctica de la Universidad Tecnológica de Pereira (Colombia). Investigadora del Grupo BIOEDUQ. https://orcid.org/0000-0001-9177-4332 